\title{
Pulse Transit Time as a Measure of Arousal and Respiratory Effort in Children with Sleep-Disordered Breathing
}

\author{
ELIOT S. KATZ, JANITA LUTZ, CHERYL BLACK, AND CAROLE L. MARCUS \\ The Eudowood Division of Pediatric Respiratory Sciences, Johns Hopkins University, Baltimore, \\ Maryland, U.S.A.
}

\begin{abstract}
The upper airway resistance syndrome (UARS) is associated with neurobehavioral morbidity in children. The diagnostic gold standard for UARS is esophageal manometry. However, this is invasive. Furthermore, upper airway obstructive events in sleeping children frequently terminate without visible electrocortical (EEG) arousal. The pulse transit time (PTT) is a noninvasive marker of blood pressure and, therefore, subcortical arousal. Blood pressure elevation, associated with respiratory arousal from sleep, results in a drop in the PTT. We hypothesized that: 1) the PTT is a more sensitive measure of respiratory arousal than EEG; and 2) the PTT arousal index can distinguish children with UARS from those with primary snoring. Polysomnography, including esophageal manometry and PTT, was measured prospectively in 24 symptomatic children and 10 normal controls. Apnea, hypopnea, and respiratory effort-related arousal events terminated in a PTT arousal $91 \%, 83 \%$, and $80 \%$ of the time, and in an EEG arousal in 55\%, 51\%, and 43\% (all $p<0.05$ ), respectively. The PTT arousal index was significantly greater in children with UARS $(6.8$ events/h) than primary snoring $(2.2$
\end{abstract}

\section{ABSTRACT}

events/h) $(p<0.05)$. We conclude that, in children, PTT arousals are a more sensitive measure of obstructive events than visible EEG arousals. (Pediatr Res 53: 580-588, 2003)

\section{AI, apnea index}

\section{Abbreviations}

AHI, apnea/hypopnea index

EMG, electromyogram

MTA, moving time average

OSAS, obstructive sleep apnea syndrome

$\mathbf{P}_{\text {es}}$, esophageal pressure

$\mathrm{PeTCO}_{2}$, end-tidal carbon dioxide

PTT, pulse transit time

PS, primary snoring

PSG, polysomnogram

RERA, respiratory effort-related arousal

$\mathrm{SaO}_{2}$, arterial oxygen saturation

TST, total sleep time

UARS, upper airway resistance syndrome
The OSAS in children is characterized by episodic upper airway obstruction that may be associated with hypoxemia, hypercarbia, and arousal. Some children exhibit recurrent episodes of partial airway obstruction, resulting in respiratory arousal in the absence of frank apnea, hypopnea, or gas exchange abnormalities, termed the UARS $(1,2)$. The significance of the UARS phenotype in children is uncertain insofar as there is no consensus on the definition of respiratory arousal and the diagnostic criteria for UARS. Both OSAS and UARS may be characterized by neurobehavioral consequences of

Received February 4, 2002; accepted October 4, 2002.

Correspondence: Carole L. Marcus, MBBCh, Division of Pediatric Pulmonology, Park 316, Johns Hopkins Hospital, 600 N. Wolfe Street, Baltimore, MD 21287-2533, U.S.A.; e-mail: cmarcus2@jhmi.edu

Supported by NIH/National Center for Research Resources Grant M01-RR00052 to the Johns Hopkins University School of Medicine (C.L.M.), and National Heart, Lung, and Blood Institute Grant HL58585 (C.L.M.). E.S.K. was supported by an American Lung Association Research of Maryland Grant.

DOI: 10.1203/01.PDR.0000057206.14698.47 sleep fragmentation such as aggressive behavior, learning disabilities, hypersomnolence, and attention deficit/hyperactivity (1). Early recognition of UARS is important insofar as treatment with adenotonsillectomy or continuous positive airway pressure is effective (1). The gold standard for diagnosing UARS is esophageal manometry to identify RERA. However, this is invasive. Furthermore, airway obstructions in sleeping children terminate without visible EEG arousals about $50 \%$ of the time (3).

The PTT is a novel measure of respiratory effort and arousal that has shown promise in the diagnosis of sleep-disordered breathing in adults $(4,5)$. The PTT is the interval between the R-wave of the ECG and the arrival of the photoplethysmographic pulse at the finger (6). The travel time of the pulse wave is inversely proportional to arterial wall stiffness, which is determined by blood pressure (7). Therefore, the PTT is a noninvasive index that is inversely related to blood pressure.

The utility of the PTT as a diagnostic tool in sleepdisordered breathing stems from alterations in blood pressure 
patterns associated with increased respiratory effort and respiratory arousal from sleep. The pulsus paradoxus is an exaggerated inspiratory decline in blood pressure that occurs with airway obstruction (8). This results in an increase in PTT. Thus, PTT provides a noninvasive assessment of respiratory effort (9). In addition, arousal at the termination of an obstructive event leads to a marked, transient increase in blood pressure (10). This results in a decrease in the PTT. Thus, PTT can be used to evaluate arousal from sleep. So-called autonomic or subcortical arousals have been described in the literature, whereby autonomic changes, such as increases in blood pressure or heart rate, occur even in the absence of visible EEG changes $(4,5,11,12)$. The PTT can also identify these subtle arousals (12) and is, thus, a marker of autonomic (subcortical) arousal. On the basis of these "subcortical" respiratory arousals, the PTT has been used to classify the presence and severity of OSAS in adults (4). The role of PTT in the diagnosis of sleep-disordered breathing in children has not been established.

We hypothesized that 1) the PTT as a measure of subcortical arousal would be a more sensitive indicator of respiratory arousal than visible EEG frequency shifts; and 2) the PTT arousal index could distinguish children with UARS from those with PS without esophageal manometry. Finally, we sought to evaluate esophageal manometry in control children who were at the peak age for sleep-disordered breathing.

\section{METHODS}

We prospectively evaluated the PTT during a comprehensive overnight PSG in patients with sleep-disordered breathing compared with a control group.

Subjects. Children with symptoms of sleep-disordered breathing $(n=24)$ between 2 and 16 y of age were recruited from the Sleep Disorders Clinic at the Johns Hopkins Hospital. Three groups of symptomatic patients were recruited: 1) children with a previous overnight PSG demonstrating OSAS who agreed to repeat polysomnography with esophageal manometry $(n=5), 2)$ children who were suspected to have UARS based on a previous PSG showing increased EEG arousals $(>10$ events/h) in the absence of gas exchange abnormalities ( $n=$ 14); and 3) children with a previously normal overnight PSG who subsequently reported worsening symptoms $(n=5)$. None of the symptomatic patients had a history of heart disease or diabetes and none were on medications known to affect cardiovascular functioning. In addition, a control group of children was recruited from the community $(n=10)$ who fulfilled all of the following: 1) no history of snoring; 2) no significant medical conditions, including heart disease; 3 ) normal physical examination; and 4) taking no medications.

All patients underwent a complete history and physical examination focusing on clinical evidence of OSAS and possible etiologic factors. Patients with conditions known to affect sleep or breathing such as craniofacial dysmorphism, mental retardation, cerebral palsy, and previous otolaryngologic surgery and those taking medications known to affect sleep were excluded. Signed, informed consent was obtained from the parent and assent from children $>5 \mathrm{y}$ of age. The study was approved by the institutional review board at our hospital.

Polysomnography. Each subject underwent an overnight PSG in our sleep laboratory, including continuous measurement of esophageal manometry and beat-to-beat PTT. Children were accompanied by a parent throughout the night. Studies were scored using standard pediatric techniques $(13,14)$. Details of our PSG montage and event definitions have been published elsewhere (15). In brief, EEG (C3-A2, C4-A1, O1A2); right and left electrooculogram; submental EMG; tibial EMG; ECG; chest and abdominal wall motion by respiratory inductance plethysmography (Respitrace, Ambulatory Monitoring, Inc., Ardsley, NY, U.S.A.); oronasal airflow (threepronged thermistor); $\mathrm{PETCO}_{2}$, measured at the nose by infrared capnometry (Nellcor N-1000, Van Nuys, CA, U.S.A); $\mathrm{SaO}_{2}$ by pulse oximetry (Nellcor N-1000); and oximeter waveform were measured. Subjects were also monitored and recorded on videotape, using an infrared video camera, and were continuously observed by a polysomnography technician. The studies were performed with a computerized system (Alice 3, Healthdyne, Marietta, GA, U.S.A.) and a Windaq data acquisition system (Dataq Instruments, Akron, OH, U.S.A.).

An obstructive apnea was defined as the presence of chest/ abdominal wall motion associated with a reduction in the thermistor tracing $\geq 80 \%$ of baseline, a loss of $\mathrm{PETCO}_{2}$ waveform, $\mathrm{P}_{\mathrm{es}}$ swings, and paradoxical respiratory efforts lasting the duration of two or more breaths. An obstructive hypopnea was defined similarly, except that the reduction in thermistor flow was $50-80 \%$ of baseline, and was associated with a drop in $\mathrm{SaO}_{2} \geq 4 \%$ and/or arousal. Apneas were considered mixed if they had both a central and obstructive component. All obstructive events were validated with esophageal manometry by demonstrating large negative pressure excursions during the event that terminated by a sudden change in pressure to a less negative level at the end of the event. The AI was defined as the total number of obstructive and mixed apneas per hour of TST. The AHI was defined as the total number of obstructive apneas, mixed apneas, and hypopneas per hour of TST. Microarousals were identified according to the definition of the American Sleep Disorders Association (16). The EEG arousal index was defined as the number of arousals and awakenings per hour of TST. RERA were defined as graded increases in $\mathrm{P}_{\mathrm{es}}$ lasting at least $10 \mathrm{~s}$ and ending in an abrupt $\mathrm{P}_{\mathrm{es}}$ reversal (17). The RERA index was defined as the number of RERA per hour of TST. The obstructed event index was defined as the total number of obstructive apneas, obstructive hypopneas, mixed apneas, and RERA per hour of TST.

Although strict PSG diagnostic criteria have not been established for OSAS in children, a PSG classification based on our clinical experience and review of the literature was made using the following general guidelines:

1. $P S$ : This classification required fulfillment of all five of the following PSG criteria: i) snoring; ii) $\mathrm{AI} \leq 1 / \mathrm{h}$; iii) $\mathrm{SaO}_{2}$ nadir $\geq 92 \%$; iv) Peak $\mathrm{PeTCO}_{2} \leq 53 \mathrm{~mm} \mathrm{Hg}$ and duration of hypoventilation $\left(\mathrm{PeTCO}_{2} \geq 50 \mathrm{~mm} \mathrm{Hg}\right)<10 \% \mathrm{TST}$; and v) RERA index $<1 / \mathrm{h}$.

2. UARS: Diagnosis of UARS required fulfillment of all four of the following PSG criteria: i) $\mathrm{AI} \leq 1 / \mathrm{h}$; ii) $\mathrm{SaO}_{2}$ nadir 
$\geq 92 \%$; iii) peak $\mathrm{PETCO}_{2} \leq 53 \mathrm{~mm} \mathrm{Hg}$ and duration of hypoventilation $<10 \%$ TST; iv) RERA index $\geq 1 / \mathrm{h}$.

3. OSAS: Diagnosis of OSAS required fulfillment of the following: $\mathrm{AI} \geq 1 / \mathrm{h}$.

Subjects who did not fulfill all of the aforementioned requirements were not included in the study. The AI rather than AHI was used in our classification criteria insofar as normative data for hypopneas in young children have not been reported (13).

Esophageal manometry. A 6-french water-filled catheter (Corpak Medsystems, Wheeling, IL, U.S.A.) was placed transnasally to the lower third of the esophagus. A bias flow of 2 $\mathrm{mL} / \mathrm{h}$ was infused through the catheter and attached to a manometer for continuous $\mathrm{P}_{\mathrm{es}}$ measurements. $\mathrm{P}_{\mathrm{es}}$ waveforms were recorded using Windaq and Alice software simultaneously. The recordings were also reviewed manually to eliminate artifact. Inspiratory peaks and troughs were determined using Advanced CODAS software (Dataq Instruments) and exported into an Excel spreadsheet (Microsoft Corp., Seattle, WA, U.S.A.). The maximum inspiratory decline in $\mathrm{P}_{\mathrm{es}}\left(\Delta \mathrm{P}_{\mathrm{es}}\right)$ was determined for each breath as a measure of respiratory effort. For each patient, the average $\Delta \mathrm{P}_{\text {es }}$, peak $\Delta \mathrm{P}_{\text {es }}$, and percentage of breaths with a $\Delta \mathrm{P}_{\mathrm{es}}$ more negative than $-10 \mathrm{~cm}$ $\mathrm{H}_{2} \mathrm{O}$ was calculated.

$\boldsymbol{P T T}$. The PTT was measured on a beat-by-beat basis using a commercially available device derived from an Ohmeda oximeter and ECG (Stowood Scientific Instruments, Oxford, U.K.). The PTT was measured as the interval between the $\mathrm{R}$-wave of the QRS complex to a threshold on the pulse waveform representing $25 \%$ of its height. The ECG and oximeter waveforms were acquired at $500 \mathrm{~Hz}$ (2-ms sample period). The PTT was stored for display using a Windaq data acquisition system and Alice software simultaneously. Tracings were carefully analyzed for artifacts caused by inadequate oximeter pulse waveform or ECG. Only artifact-free PTT waveforms were used for analysis. Inspiratory peaks and troughs were determined using Advanced CODAS software and exported into an Excel spreadsheet. As a measure of respiratory effort
(9), the inspiratory rise in PTT ( $\triangle \mathrm{PTT})$ was measured for each breath and averaged over the night. A correlation coefficient was calculated between the mean overnight $\Delta$ PTT and the mean overnight $\Delta \mathrm{P}_{\mathrm{es}}$.

PTT arousals were derived from a 4-second MTA of the raw PTT signal to eliminate respiratory variation using Advanced CODAS software. A PTT arousal was then defined as a decline in the averaged signal of $\geq 15 \mathrm{~ms}$, lasting at least $5 \mathrm{~s}$ (4). The PTT arousal index was defined as the number of PTT arousals per hour of TST.

Data analysis. All data are expressed as mean $\pm \mathrm{SD}$. The group means were compared using an ANOVA analysis. Multiple comparisons between groups were tested by the StudentNewman-Keuls method. A value of $p<0.05$ was considered statistically significant. The overall percentages of obstructive events associated with PTT and EEG arousals were compared using $\chi^{2}$ analysis.

\section{RESULTS}

Demographic and polysomnographic data for our subject population are summarized in Table 1. Based on the comprehensive PSG in this study, there were eight patients with PS, five with UARS, and 11 with OSAS. All normal control subjects were confirmed as normal based on the PSG. No patients were excluded from the study because of failure to strictly fit into classification criteria. Children with PS and UARS were significantly older than those with OSAS or control patients $(p<0.05)$. Symptomatic children had a higher percent predicted BMI (18) and were more likely to be obese than control patients $(p<0.05)$. In control subjects, the correlation coefficients between age and the mean $\Delta \mathrm{P}_{\mathrm{es}}$, mean $\Delta$ PTT, and PTT index was $-0.11,0.14$, and --0.18 , respectively (all NS). Also, in control subjects, the correlation coefficient between percentage predicted $\mathrm{BMI}$ and the mean $\Delta \mathrm{P}_{\mathrm{es}}$, mean $\Delta$ PTT, and PTT index was $0.05,0.22$, and 0.15 (all NS), respectively. The sleep architecture variables, including sleep efficiency, percentage slow wave sleep, and percentage rapid

Table 1. Demographic and polysomnographic data of study subjects

\begin{tabular}{|c|c|c|c|c|}
\hline & Normal & PS & UARS & OSAS \\
\hline No. & 10 & 8 & 5 & 11 \\
\hline Age (y) & $6.2 \pm 3$ & $9.7 \pm 3 *$ & $9.9 \pm 2 *$ & $5.4 \pm 3+t$ \\
\hline Range & $(2-11)$ & $(3-16)$ & $(8-14)$ & $(2-10)$ \\
\hline BMI $(\%$ predicted $)$ & $46 \pm 21$ & $69 \pm 34^{*}$ & $56 \pm 30^{*}$ & $59 \pm 28 *$ \\
\hline Obese (no. $>95$ th percentile for BMI) & 0 & 5 & 1 & 2 \\
\hline Sleep efficiency $(\%)$ & $85 \pm 8$ & $81 \pm 10$ & $78 \pm 17$ & $82 \pm 11$ \\
\hline $\mathrm{AI}$ (events/h) & $0 \pm 0$ & $0.2 \pm 0.1$ & $0.1 \pm 0.1$ & $7.3 \pm 10 * \dagger+$ \\
\hline AHI (events/h) & $0 \pm 0$ & $0.3 \pm 0.2$ & $0.7 \pm 0.5$ & $10.6 \pm 11 *+t$ \\
\hline $\mathrm{SaO}_{2}$ nadir $(\%)$ & $96 \pm 1$ & $94 \pm 3$ & $94 \pm 4$ & $90 \pm 4^{*}$ \\
\hline Peak $\mathrm{PeTCO}_{2}(\mathrm{~mm} \mathrm{Hg})$ & $46 \pm 2$ & $48 \pm 3$ & $46 \pm 3$ & $52 \pm 4 * t$ \\
\hline EEG arousal index (events/h) & $7.5 \pm 2$ & $8.2 \pm 2$ & $11 \pm 3 * \dagger$ & $10.5 \pm 4^{* \dagger}$ \\
\hline RERA index (events/h) & $0 \pm 0$ & $0.2 \pm 0.2$ & $4.6 \pm 2.2 * \dagger$ & $5.9 \pm 12.0 * \dagger$ \\
\hline
\end{tabular}

Data are presented as mean \pm SD unless otherwise specified. BMI, body mass index; SWS, slow wave sleep; REM, rapid eye movement.

* Significant difference compared with normals $(p<0.05)$.

$\dagger$ Significant difference compared with PS $(p<0.05)$.

$\ddagger$ Significant difference compared with UARS $(p<0.05)$. 
eye movement sleep, were not significantly different between the groups. Children with UARS and OSAS as a group had a significantly higher EEG arousal index than those with PS or controls $(p<0.05)$, but there was considerable overlap.

An example of the raw and MTA PTT signal during REM sleep with and without obstruction is shown in Figure 1. Examples of PTT arousals associated with EEG arousals in a UARS patient are shown in Figure 2, and PTT arousals without EEG arousals in an OSAS patient are shown in Figure 3.

\section{Respiratory Effort}

Plots of the mean $\Delta \mathrm{P}_{\mathrm{es}}$ and mean $\Delta \mathrm{PTT}$ are shown in Figure 4. $\Delta \mathrm{P}_{\mathrm{es}}$ was significantly increased in all three symptomatic groups compared with controls $(p<0.05)$. Children with OSAS had a significantly greater $\Delta \mathrm{P}_{\text {es }}$ than both PS subjects ( $p$ $<0.05)$ and UARS patients $(p<0.05)$. However, there was no significant difference in the mean $\Delta \mathrm{P}_{\text {es }}$ between patients with PS and UARS.

The mean $\triangle$ PTT (a measure of respiratory effort) was also significantly increased in the symptomatic patients compared with controls $(p<0.05)$ (Fig. 4). Children with OSAS did have a significantly greater $\Delta$ PTT than PS subjects $(p<0.05)$ but not UARS subjects. However, there was no difference in the mean $\Delta$ PTT between patients with PS and UARS. The correlation coefficient between the mean $\Delta \mathrm{P}_{\mathrm{es}}$ and the mean $\Delta \mathrm{PTT}$ was $0.38(p<0.05)$.

Control subjects had a mean $\Delta \mathrm{P}_{\mathrm{es}}$ of $-7.9 \pm 2 \mathrm{~cm} \mathrm{H}_{2} \mathrm{O}$ (range, -5.9 to -11.9 ), a peak $\Delta \mathrm{P}_{\mathrm{es}}$ of $-12 \pm 3 \mathrm{~cm} \mathrm{H}_{2} \mathrm{O}$ (range, -9 to $-19 \mathrm{~cm} \mathrm{H}_{2} \mathrm{O}$ ), and had a $\Delta \mathrm{P}_{\mathrm{es}}$ more negative than $-10 \mathrm{~cm} \mathrm{H}_{2} \mathrm{O}$ for $8 \pm 12 \%$ of breaths (range, 3-61\%). Two of the normal subjects had a $\Delta \mathrm{P}_{\mathrm{es}}$ more negative than -10 $\mathrm{cm} \mathrm{H}_{2} \mathrm{O}$ for $>10 \%$ of the night $(21 \%$ and $61 \%)$. No control subjects had any RERA.

Respiratory arousal. PTT arousals were significantly more common than EEG arousals during all categories of obstructive events $(p<0.05)$ (Table 2$)$. In addition, PTT arousals were more likely to be associated with an obstructive event than EEG arousals $(p<0.05)$ (Table 3$)$. Plots of the obstructive event index and the PTT arousal index are shown in Figure 5. Patients with UARS and OSAS had a significantly greater obstructive event index than control and PS patients $(p<0.05)$, as expected from our classification definitions. Children with UARS and OSAS had significantly greater PTT arousal indices than those with PS $(p<0.05)$. The PTT arousal index was not significantly different in children with PS compared with controls, nor between those with UARS and OSAS. The use of a threshold level of the PTT arousal index to classify symptomatic subjects with an AI $<1$ (those with an AI $>1$ were classified as having OSAS) was evaluated insofar as our primary aim was to determine the utility of the PTT in distinguishing PS from UARS. In these patients, empirically setting a PTT arousal index threshold of four events per hour as indicative of UARS would have identified all children with UARS, and only misclassified one PS patient as having UARS. Using this threshold in this small sample, the sensitivity was $100 \%$, the specificity was $88 \%$, and the positive predictive value was $83 \%$.
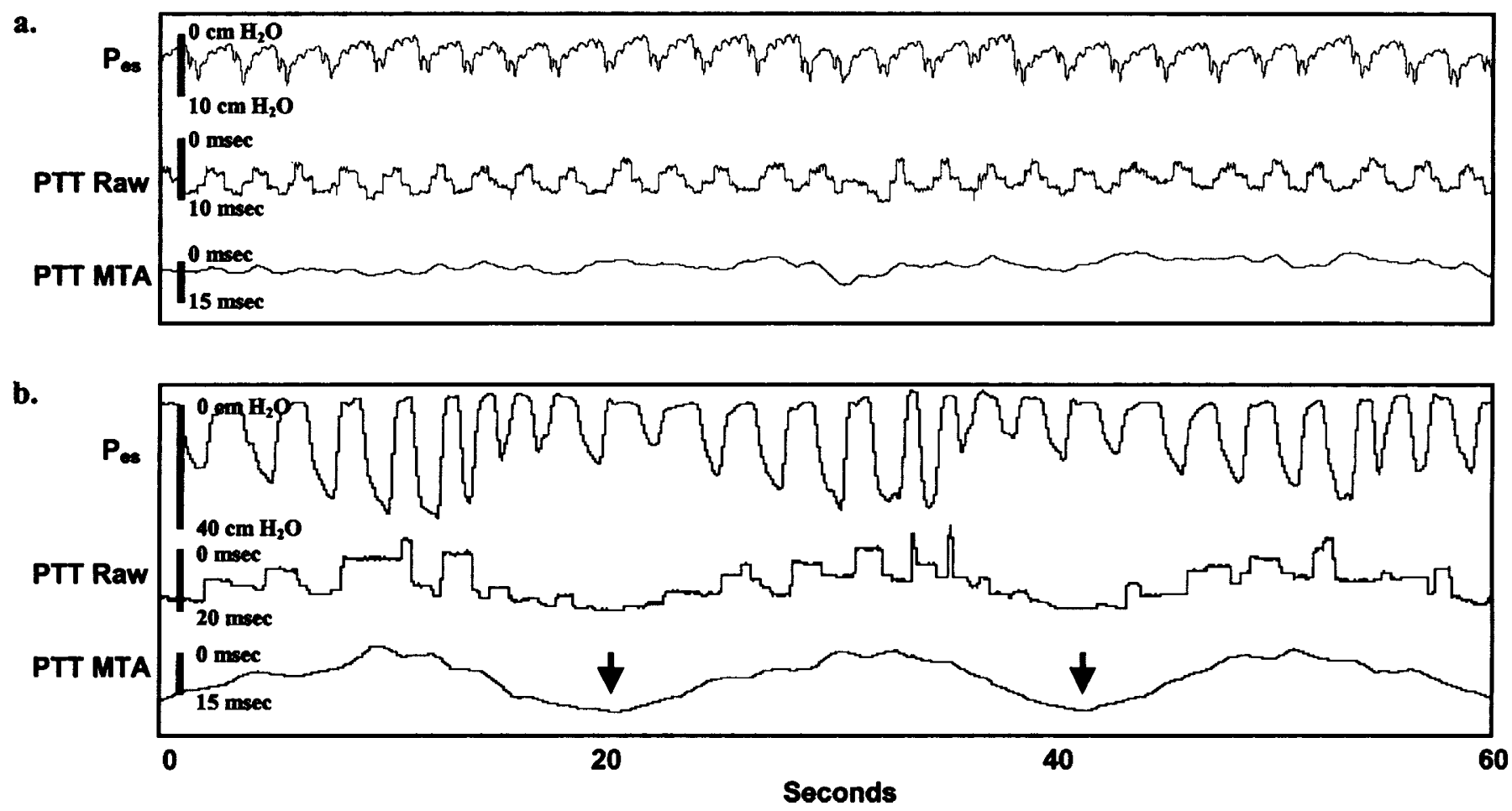

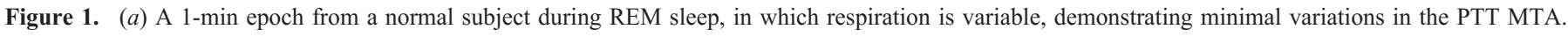

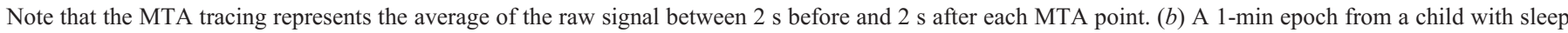

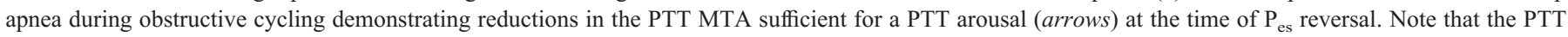
arousal often occurs 5-10 s after the resumption of breathing, in keeping with the time course of the blood pressure elevation during respiratory arousal. 


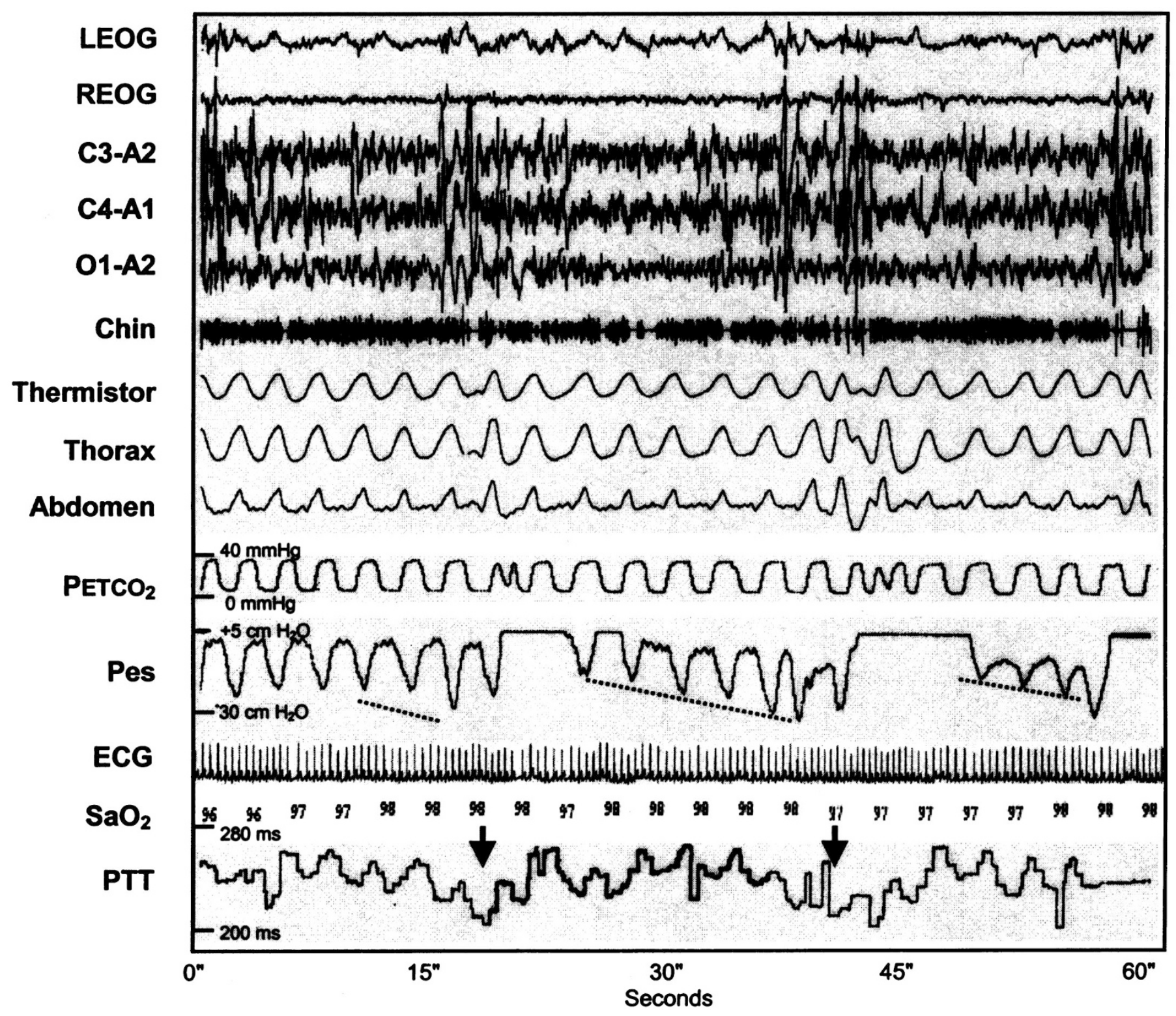

Figure 2. Example of PTT and EEG arousals after RERA. A 60-s epoch from a 13-y-old male with the UARS is shown. There are a series of $P_{\text {es }}$ crescendos but only minimal changes in the thermistor and $\mathrm{PETCO}_{2}$ channels. These events terminate in simultaneous EEG and PTT arousals. Inspiration is upward on thermistor tracing. Expiration is upward for $\mathrm{PETCO}_{2}$. The $\mathrm{P}_{\mathrm{es}}$ channel has been truncated above $+5 \mathrm{~cm} \mathrm{H} \mathrm{H}_{2} \mathrm{O}$ for ease of viewing. $L E O G$, left electrooculogram; REOG, right electrooculogram; C3-A2, C4-A1, O1-A2, EEG channels. Dotted lines highlight crescendos in $\mathrm{P}_{\mathrm{es}}$. Solid arrows indicate PTT arousals.

\section{DISCUSSION}

We prospectively measured the PTT during a comprehensive overnight PSG in children with symptoms of sleepdisordered breathing compared with a normal control group. PTT arousals often occurred in the absence of EEG arousals during apnea, hypopnea, and RERA obstructive events. The PTT arousal index was significantly higher in children with UARS versus those with PS, consistent with the elevated obstructive event index observed in the former condition. Thus, PTT arousals were useful in distinguishing UARS from PS. However, as a measure of respiratory effort, the mean inspiratory $\triangle$ PTT was not significantly different between children with PS and UARS, consistent with the observed increased $\Delta \mathrm{P}_{\mathrm{es}}$ in both conditions. Thus, although the mean inspiratory $\triangle$ PTT reflects work of breathing, this feature did not further facilitate classification of disease groups.

Although $3-12 \%$ of children have habitual snoring, only $1-3 \%$ have OSAS (19-21). Snoring in children without apnea, hypopnea, increased EEG arousals, or gas exchange abnormalities has been termed PS (22). Although few data exist regarding the consequences of PS, it is widely considered comparatively benign relative to UARS or OSAS (15). However, distinguishing UARS from PS in children is complicated by the fact that obstructive events are often not accompanied by EEG evidence of arousal (3). Furthermore, many spontaneous EEG arousals are not associated with respiratory events. Thus, UARS may go unrecognized unless esophageal manometry, a relatively invasive measure of respiratory effort, is used (2). 


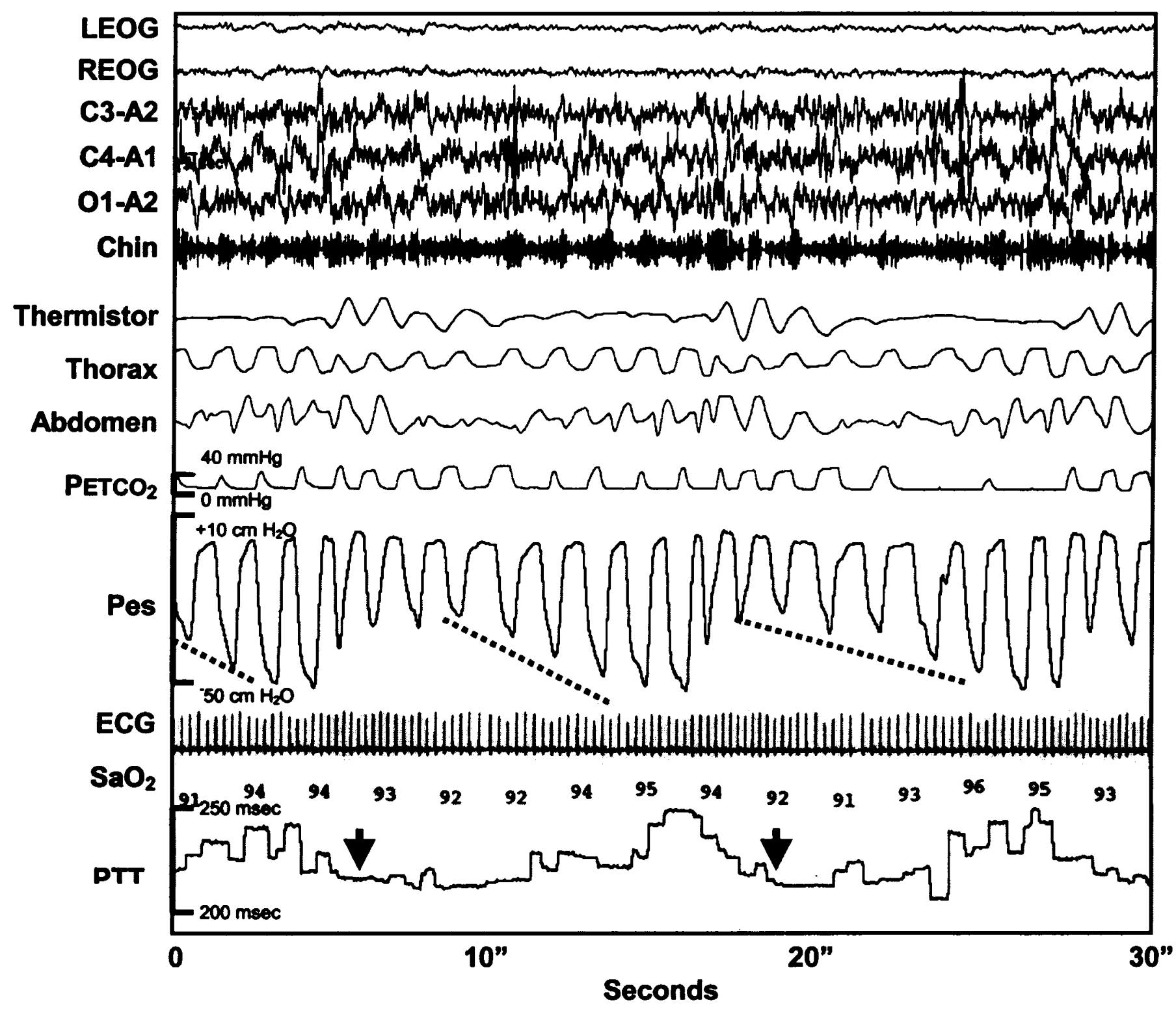

Figure 3. Example of PTT arousals without EEG arousals after obstructive apneas. A 30-s epoch from a 7-y-old male with the OSAS is shown. Obstructions

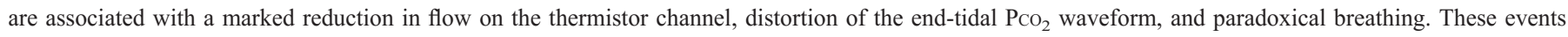
are associated with $\mathrm{P}_{\mathrm{es}}$ crescendos and terminate in PTT arousals. However, there are no visible EEG arousals. Inspiration is upward on thermistor tracing. Expiration is upward for $\mathrm{PETCO}_{2}$. LEOG, left electrooculogram; REOG, right electrooculogram; C3-A2, C4-A1, O1-A2, EEG channels. Dotted lines highlight crescendos in $\mathrm{P}_{\mathrm{es}}$. Solid arrows indicate PTT arousals.

Although accurate, $\mathrm{P}_{\mathrm{es}}$ recording is uncomfortable and laborintensive. In addition, obstructive and central apnea indices may be slightly lower when an esophageal probe is in place (23). Thus, a noninvasive marker of respiratory arousal would be of considerable utility in the diagnosis of UARS.

In adults, the PTT arousal index has been used as a measure of OSAS (9). This is the first study to evaluate the PTT in children with sleep-disordered breathing. We chose to use the definition of PTT arousal that has been validated in adults (4). Although declines in PTT $<15 \mathrm{~ms}$, or event durations $<5 \mathrm{~s}$ may also indicate arousal (12), the number of false-positive events would also increase. Overall, obstructive events were accompanied by PTT arousals $83 \%$ of the time, compared with only $48 \%$ for EEG arousals (Table 2 ).
In addition, in our sample, the positive predictive value of a PTT arousal or EEG arousal being associated with an obstructive event was $65 \%$ and $26 \%$, respectively (Table 3 ). Some of the false-positive PTT arousals may, in fact, represent spontaneous arousals. Our data indicate that the PTT arousal index in children with UARS and OSAS is elevated compared with PS. Because our selected sample of OSAS patients included those with mild disease, there was considerable overlap in the PTT arousal index between children with PS and OSAS. However, among patients with an AI $<1$, setting a PTT arousal index threshold of four events per hour would have identified all children with UARS, and would have only misclassified one PS patient as having UARS. 

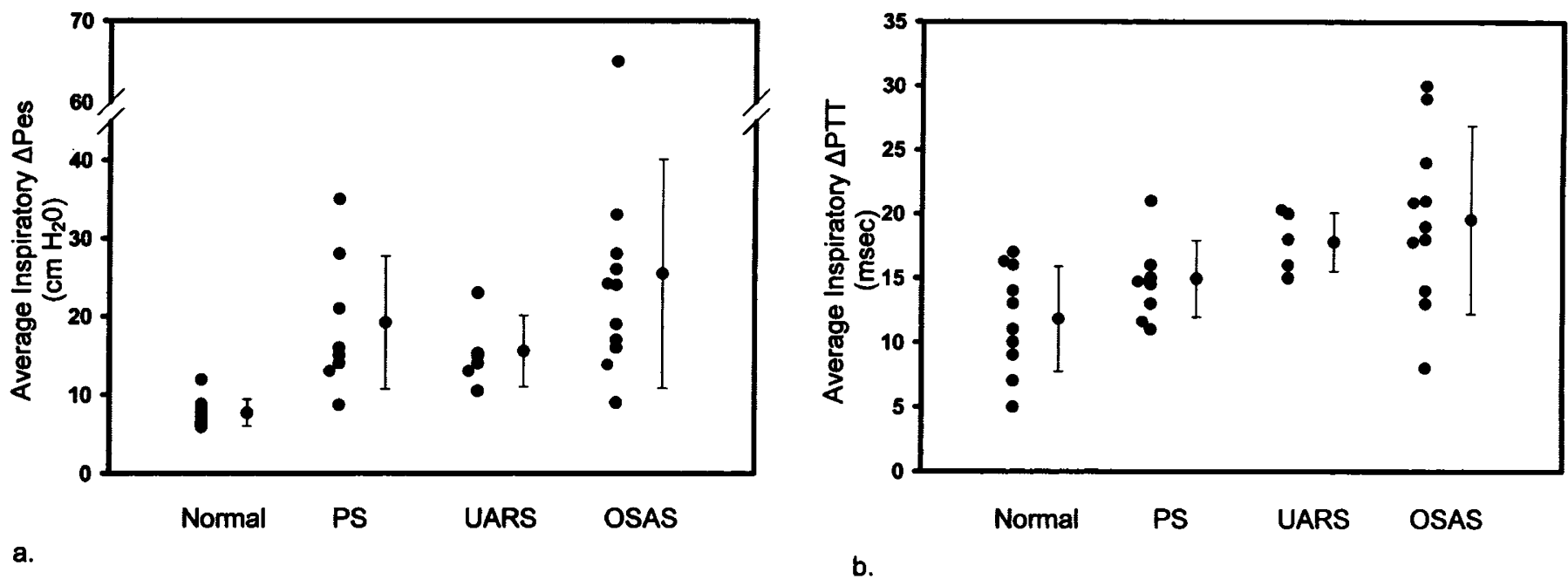

Figure 4. Plots of the overnight average inspiratory $\Delta \mathrm{P}_{\mathrm{es}}(a)$ and inspiratory $\Delta \mathrm{PTT}(b)$ for 24 children with sleep-disordered breathing and 10 controls. Normal control subjects had a significantly lower $\Delta \mathrm{P}_{\text {es }}$ and $\Delta$ PTT than patients with PS, UARS, and OSAS $(p<0.05)$. Children with OSAS had a significantly greater $\Delta \mathrm{P}_{\mathrm{es}}$ than those with UARS or PS $(p<0.05)$. Children with OSAS had a significantly greater $\Delta \mathrm{PTT}$ than those with PS $(p<0.05)$.

Table 2. PTT and EEG arousals during obstructive events

\begin{tabular}{lcccc}
\hline & & & & $\begin{array}{c}\text { All } \\
\text { obstructive } \\
\text { events }\end{array}$ \\
& RERA & Hypopnea & Apnea & 1121 \\
Events (No.) & 512 & 375 & 234 & $48^{*}$ \\
EEG arousals (\%) & 43 & 51 & 55 & $83^{*}$ \\
PTT arousals (\%) & 80 & 83 & 91 & \\
\hline
\end{tabular}

$* p<0.05$ for EEG arousals $v$. PTT arousals.

Table 3. Obstructive events during PTT and EEG arousals

\begin{tabular}{lcc}
\hline & & PTT \\
& EEG Arousal & Arousal \\
\hline N (events) & 2051 & 1442 \\
RERA (\%) & 11 & 28 \\
Hypopnea (\%) & 9 & 22 \\
Apnea (\%) & 6 & 15 \\
All obstructive events (\%) & $26^{*}$ & $65^{*}$ \\
\hline
\end{tabular}

$* p<0.05$ for EEG arousals $v s$. PTT arousals.

RERA, respiratory effort-related arousal; EEG, electroencephalogram; PTT, pulse transit time.

The PTT arousal is thought to indicate a subcortical or autonomic arousal response, whereas the EEG arousal represents a cortical response. In the current study, PTT arousals occurred more frequently than visible EEG arousals $(83 \%$ versus $48 \%$, respectively) at the termination of obstructive events (Table 2). Frank apneas were more likely to be associated with a PTT arousal than RERA (91\% versus $80 \%)$. This corroborates previous work showing that children may manifest respiratory movement arousals in the absence of EEG arousals (24). It is possible that these subcortical responses contribute to the autonomic and neurobehavioral sequelae of childhood OSAS.

Previous work has demonstrated an excellent correlation between inspiratory $\Delta \mathrm{PTT}$ and $\Delta \mathrm{P}_{\mathrm{es}}$ in OSAS patients during brief alterations in continuous positive airway pressure (9). An elevation in average inspiratory $\triangle \mathrm{PTT}$ has been reported in adults with severe OSAS versus mild to moderate OSAS, although the specificity is poor (4). Our data indicate that the overnight average inspiratory $\Delta \mathrm{PTT}$ is significantly correlated with the average $\Delta \mathrm{P}_{\mathrm{es}}$ and is elevated in children with PS, UARS, and OSAS compared with a control group. The frequent respiratory arousals observed in UARS and OSAS contributed to the lowering of the average inspiratory $\Delta \mathrm{P}_{\mathrm{es}}$ and $\Delta$ PTT toward the levels seen in PS. Thus, the average inspiratory $\triangle \mathrm{PTT}$ as a measure of respiratory effort during a PSG provided no additional information to support the distinction between UARS and PS. However, as a measure of respiratory effort, the inspiratory $\triangle$ PTT may be useful as a marker of increased work of breathing and may facilitate distinguishing central from obstructive apneic events during polysomnography (25).

Although esophageal manometry is considered the definitive measure of UARS, few data on $\Delta \mathrm{P}_{\mathrm{es}}$ for normal children in this age group have been reported. Some investigators have considered $\Delta \mathrm{P}_{\text {es }}$ swings of -8 to $-14 \mathrm{~cm} \mathrm{H}_{2} \mathrm{O}$ as normal (26), whereas others suggested that normal children spend $<10 \%$ of the night with inspiratory $\mathrm{P}_{\text {es }}$ swings more negative than -10 $\mathrm{cm} \mathrm{H}_{2} \mathrm{O}$ (2). However, our data showed that in two control patients the $\Delta \mathrm{P}_{\mathrm{es}}$ was more negative than $-10 \mathrm{~cm} \mathrm{H}_{2} \mathrm{O}$ for $21 \%$ and $61 \%$ of the night. Although our control sample was small, the mean $\Delta \mathrm{P}_{\mathrm{es}}$ was $-7.9 \pm 2 \mathrm{~cm} \mathrm{H}_{2} \mathrm{O}$ (range, -5.9 to -11.9 ) with a peak $\Delta \mathrm{P}_{\mathrm{es}}$ range from -9 to $-19 \mathrm{~cm} \mathrm{H}_{2} \mathrm{O}$. Thus, normative data from nonsnoring controls revealed more variability in respiratory effort than previously reported.

The PTT has some theoretical and practical limitations. By definition, a PTT measure is only available once per cardiac cycle, necessarily resulting in an undersampling error (9). This is especially problematic in children as they have relatively higher respiratory rates. The undersampling of individual breaths is likely to have had its most profound effect on the use of the mean inspiratory $\triangle \mathrm{PTT}$ as a measure of respiratory effort. However, the use of continuous $\mathrm{P}_{\mathrm{es}}$ monitoring supports the conclusion from our mean inspiratory $\triangle \mathrm{PTT}$ data that respiratory effort was not significantly different between UARS and PS (Fig. 3). The physiologic variability in respiratory effort 

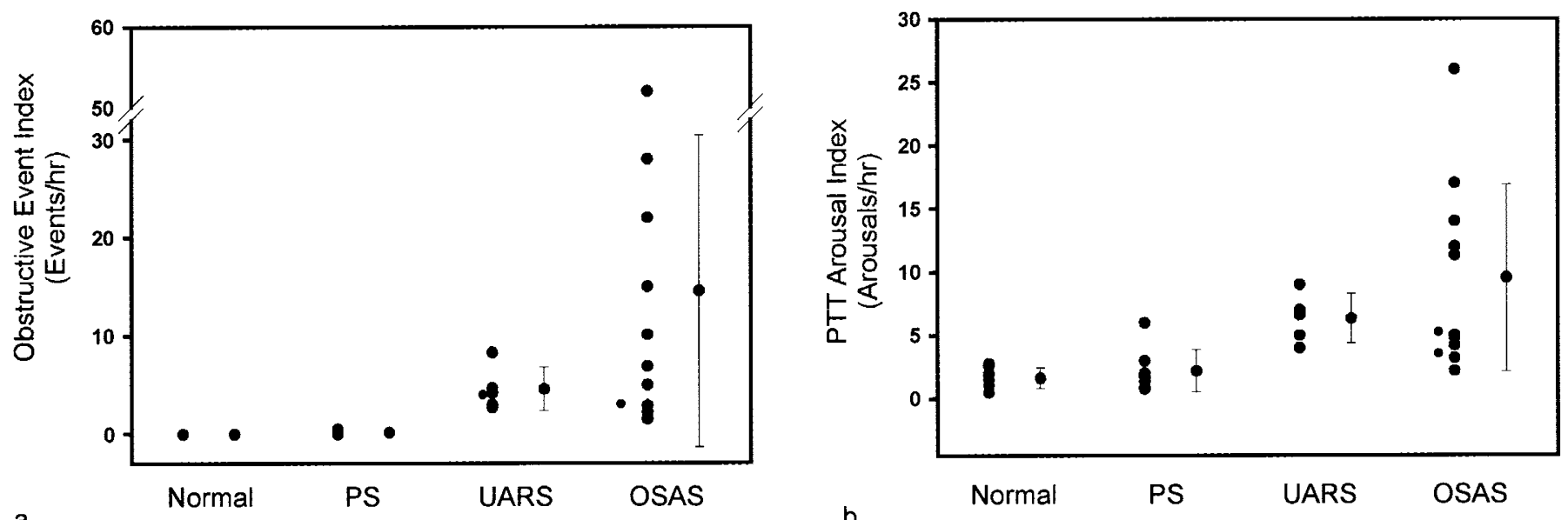

Figure 5. Plots of the overnight obstructive event index $(a)$ and PTT arousal index $(b)$ for 24 children with sleep-disordered breathing and 10 controls. Patients with UARS and OSAS had a significantly higher obstructive event index and PTT arousal index than patients with PS $(p<0.05)$ or controls $(p<0.05)$.

and heart rate observed during REM sleep may make interpretation of the $\triangle \mathrm{PTT}$ and the MTA PTT difficult to distinguish from obstruction and arousal. Another source of variability in the measurement of the PTT may arise from changes in the cardiac preejection period. The PTT is comprised of the preejection period followed by the arterial travel time. This effect, however, has been shown to be small (12). Motion artifact of the oximeter may occur during movement associated with an arousal, occasionally rendering the PTT measurement invalid. More sophisticated techniques, using spectral analysis, have shown cortical changes not detected by routine visual scoring (27). Nevertheless, our data support the fact that the PTT is a more sensitive measure of arousals than visible EEG changes.

The symptomatic patients evaluated in this study were drawn from a large clinic population on the basis of worsening symptoms and an elevated EEG arousal index, and from those children with known OSAS who agreed to participate. This selection may have introduced a bias that may limit the generalizability of our findings. In addition, the patients with PS and UARS were slightly older than the OSAS and control groups. The peak age of OSAS is between 2 and 6 y of age, when the adenotonsillar tissue is at its maximal volume relative to the size of the nasopharynx (20). Children with UARS are generally diagnosed at an older age, when the neurocognitive sequelae and hypersomnolence may be more readily apparent $(1,2)$. The observation that PS patients were slightly older may reflect a referral bias. The age disparity was unlikely to affect the results insofar as the correlation between age and the PTT variables was very low. The symptomatic patients also had a higher percentage predicted BMI than did the control group. In the control subjects, there was no significant correlation between $\Delta \mathrm{P}_{\mathrm{es}}$ and percentage predicted BMI. In adults, an increased work of breathing $\left(\Delta \mathrm{P}_{\mathrm{es}}\right)$ secondary to obesity has been previously reported (28). However, our primary goal was to compare children with PS and UARS, between whom BMI and age were not significantly different.

Finally, to properly test the utility of the PTT in pediatric sleep-disordered breathing, it was necessary to accurately dis- tinguish PS from UARS. We chose esophageal manometry as our gold standard for identifying obstructive events, which represents the reference standard of respiratory effort according to the American Academy of Sleep Medicine taskforce on definitions of sleep-disordered breathing (29). Nasal cannula pressure recording has shown promise in the diagnosis of subtle, partially obstructive events (30). However, mouthbreathing and nasal secretions may limit its effectiveness in some children (30).

This is the first report on the use of the PTT in children with sleep-disordered breathing. Our data indicate that the average inspiratory $\triangle \mathrm{PTT}$ is not useful in distinguishing PS from UARS insofar as respiratory effort is increased in both conditions. However, the PTT is a sensitive indicator of respiratory arousal. Importantly, the PTT arousal index was elevated in children manifesting episodic subcortical arousals resulting from obstructive respiratory events compared with children with PS, suggesting that the PTT may be a noninvasive alternative to esophageal manometry. Further prospective study of the PTT arousal index in larger numbers of children with suspected UARS is needed to confirm the usefulness of this tool in children.

\section{REFERENCES}

1. Guilleminault C, Winkel R, Korobkin R, Simmons B 1982 Children and nocturnal snoring: evaluation of the effects of sleep related respiratory resistive load and daytime functioning. Eur Respir J 139:165-171

2. Guilleminault C, Pelayo R, Leger D, Clerk A, Bocian RCZ 1996 Recognition of sleep-disordered breathing in children. Pediatrics 98:871-882

3. McNamara F, Issa F, Sullivan C 1996 Arousal pattern following central and obstructive breathing abnormalities in infants and children. J Appl Physiol 81:2651-2657

4. Pitson DJ, Stradling JR 1998 Value of beat-to-beat blood pressure changes, detected by pulse transit time, in the management of the obstructive sleep apnoea/hypopnoea syndrome. Eur Respir J 12:685-692

5. Argod J, Pepin J, Smith RP, Levy P 2000 Comparison of esophageal pressure with pulse transit time as a measure of respiratory effort for scoring obstructive nonapneic respiratory events. Am J Respir Crit Care Med 162:87-93

6. Geddes L, Voelz MH, Babbs CF, Bourland JD, Tacker WA 1981 Pulse transit time as an indicator of arterial blood pressure. Psychophysiology 18:71-74

7. Pollak MH, Obrist PA 1983 Aortic-Radial pulse transit time and ECG Q-wave to radial pulse wave interval as indices of beat-by-beat blood pressure changes. Psychophysiology 20:21-28

8. Parsons GH, Green JF 1978 Mechanisms of pulsus paradoxus in upper airway obstruction. J Appl Physiol 45:598-603 
9. Pitson DJ, Sandell A, van den Hout R, Stradling JR 1995 Use of pulse transit time as a measure of inspiratory effort in patients with obstructive sleep apnoea. Eur Respir J 8:1669-1674

10. Somers VK, Dyken ME, Clary MP, Abboud FM 1995 Sympathetic neural mechanisms in obstructive sleep apnea. J Clin Invest 96:1897-1904

11. Bennett LS, Langford BA, Stradling JR, Davies RJO 1998 Sleep fragmentation indices as predictors of daytime sleepiness and nCPAP response in obstructive sleep apnea. Am J Respir Crit Care Med 158:778-786

12. Pitson DJ, Chhina N, Knijn S, van Herwaaden M, Stradling JR 1994 Changes in pulse transit time and pulse rate as markers of arousal from sleep in normal subjects. Clin Sci (Lond) 87:269-273

13. American Thoracic Society 1996 Standards and indications for cardiopulmonary sleep studies in children. American Thoracic Society. Am J Respir Crit Care Med 153:866-878

14. Rechtschaffen A, Kales A 1968 A Manual of Standardized Terminology: Techniques and Scoring Systems for Sleep Stages of Human Subjects. Los Angeles, UCLA Brain Information Service/Brain Research Institute

15. Marcus C, Hamer A, Loughlin G 1998 Natural history of primary snoring. Pediatr Pulmonol 26:6-11

16. Sleep Disorders Atlas Task Force of the American Sleep Disorders Association 1992 EEG arousals: scoring rules and examples: a preliminary report from the Sleep Disorder Atlas Task Force of the American Sleep Disorders Association. Sleep 15:173-184

17. Guilleminault C, Stoohs R, Clerk A, Cetel M, Maistros P 1993 A cause of excessive daytime sleepiness. The upper airway resistance syndrome. Chest 104:781-787

18. Hammer LD, Kraemer HC, Wilson DM, Ritter PL, Dombusch SM 1991 Standardized percentile curves of body-mass index for children and adolescents. Am J Dis Child 145:259-263

19. Redline S, Tishler PV, Schluchter M, Aylor J, Clark K, Graham G 1999 Risk factor for sleep-disordered breathing in children: associations with obesity, race, and respiratory problems. Am J Respir Crit Care Med 159:1527-1532
20. Ali NJ, Pitson DJ, Stradling JR 1993 Snoring, sleep disturbance and behaviour in 4-5 year olds. Arch Dis Child 68:360-366

21. Gislason T, Benediktsdottir B 1995 Snoring, apneic episodes, and nocturnal hypoxemia among children 6 months to 6 years old. Chest 107:963-966

22. American Sleep Disorders Association 1997 The International Classification of Sleep Disorders. Diagnostic and Coding Manual, Rev Ed. Rochester, MN, American Sleep Disorders Association

23. Groswasser J, Scaillon M, Rebuffat E, Simon T, De Groote A, Sottiaux M, Kahn A 2000 Naso-oesophageal probes decrease the frequency of sleep apnoeas in infants. J Sleep Res 9:193-196

24. Mograss M, Ducharme F, Brouillette R 1994 Movement/arousals. Description, classification and relationship to sleep apnea in children. Am J Respir Crit Care Med 150:1690-1696

25. Argod J, Pepin J, Levy P 1998 Differentiating obstructive and central sleep respiratory events through pulse transit time. Am J Respir Crit Care Med 158:1778-1783

26. Miyazaki S, Itasaka Y, Yamakawa K, Okawa M, Togawa K 1988 Respiratory disturbance during sleep due to adenoid-tonsillar hypertrophy. Am J Otolaryngol 10:143-149

27. Bandla HPR, Gozal D 2000 Dynamic changes in the EEG spectra during obstructive sleep apnea in children. Pediatr Pulmonol 29:359-365

28. Berg S, Hybbinette JC, Gislasson T, Ovesen J 1995 Intrathoracic pressure variations in obese habitual snorers. J Otolaryngol 24:238-241

29. American Academy of Sleep Medicine Task Force 1999 Sleep-related breathing disorders in adults: recommendations for syndrome definition and measurement techniques in clinical research. The Report of an American Academy of Sleep Medicine Task Force. Sleep 22:667-689

30. Trang H, Leske V, Gaultier C 2002 Use of nasal cannula for detecting sleep apneas and hypopneas in infants and children. Am J Respir Crit Care Med $166: 464-468$ 